\title{
Knockdown of the IncRNA SNHG8 inhibits cell growth in Epstein-Barr virus-associated gastric carcinoma
}

Jing Liu', Chunxia Yang ${ }^{1}$, Yufang Gu ${ }^{2}$, Chong Li ${ }^{1}$, Huamei Zhang ${ }^{1}$, Wenfang Zhang ${ }^{1}$, Xueqing Wang ${ }^{1}$, Nan $\mathrm{Wu}^{1}$ and Chunyan Zheng ${ }^{3^{*}}$

* Correspondence:

Chunyanzheng2017@163.com

${ }^{3}$ Department of Medical Care, Zibo Central Hospital, 54 Gongqingtuan Rd, Zibo, Shandong 255036, People's Republic of China Full list of author information is available at the end of the article

\begin{abstract}
Background: Epstein-Barr virus (EBV) infection is causatively associated with a variety of human cancers, including gastric cancer (GC), which has one of the highest mortality rates of all human cancers. Long non-coding RNAs (IncRNAs) show important regulatory roles in human GC. SNHG8 is a recently identified IncRNA that was reported to show abnormal expression pattern in GC. However, little is known of its biological function in EBV-associated GC.

Methods: We used cell viability, colony formation and cell cycle assays to investigate the roles of IncRNA SNHG8 in the cell growth of EBV-associated GC.

Results: The transcript levels of SNHG8 in the cultured EBV-associated GC cells were significantly higher in the cultured EBV-associated GC cells compared with the levels in normal human gastric mucosal cells and EBV-negative GC cells. Knockdown of SNHG8 with specific shRNAs inhibited cell proliferation and colony formation and arrested the cell cycle in the G0/G1 phase in vitro. We also found that knockdown of SNHG8 suppressed tumor growth in vivo.
\end{abstract}

Conclusions: These data indicate the pro-oncogenic potential of SNHG8 in EBVassociated GC, meaning it is a latent therapeutic target for the treatment of this type of cancer.

Keywords: SNHG8, Cell growth, shRNA, Epstein-Barr virus-associated gastric carcinoma

\section{Background}

Epstein-Barr virus (EBV) is a gamma herpes virus that infects over $90 \%$ of the world's adult population. It can exist asymptomatically in the human system for a long time [1, 2].

A number of human malignancies are reported to be associated with EBV infection, including multiple types of Burkitt's lymphoma, Hodgkin's disease, nasal natural killer/ T-cell lymphoma, nasopharyngeal carcinoma and gastric carcinoma (GC) [3-7]. EBVassociated GC constitutes almost a tenth of all GC cases, and about 75,000 new cases of EBV-associated GC occur worldwide each year [7, 8]. A recent study suggested that this $10 \%$ estimate might be too low, as $48 / 75$ GC cases in the U.S. (64\%) and $38 / 38$ in Central America (100\%) showed positive for EBV.

(c) The Author(s). 2018 Open Access This article is distributed under the terms of the Creative Commons Attribution 4.0 International License (http://creativecommons.org/licenses/by/4.0/), which permits unrestricted use, distribution, and reproduction in any medium, provided you give appropriate credit to the original author(s) and the source, provide a link to the Creative Commons license, and indicate if changes were made. The Creative Commons Public Domain Dedication waiver (http://creativecommons.org/ publicdomain/zero/1.0/) applies to the data made available in this article, unless otherwise stated. 
$\mathrm{GC}$ is the fourth most common cancer worldwide and ranks second on the cause list of cancer deaths [9]. EBV-associated GC is very difficult to treat: the complete elimination of tumor cells via surgical, radio-therapeutic and chemotherapeutic methods is challenging [10]. New therapeutic approaches are essential.

Long non-coding RNAs (lncRNAs) are currently defined as transcripts of $\geq 200$ nt but without open reading frames (ORFs) [11]. Many studies have revealed that lncRNAs have regulatory functions, including modulation of apoptosis and invasion, reprogramming of induced pluripotent stem cells, markers of cell fate, and parental imprinting [12]. A link between altered expression of lncRNAs and cancer pathogenesis has been recognized, providing new insight into the genetic and molecular mechanisms of cancer [13-15]. In the case of gastric cancer, lncRNA dysregulation is associated with larger tumors, greater tumor invasion, more widespread metastasis, and lower survival rates $[16,17]$. However, few studies have investigated lncRNAs in EBV-associated GC.

SNHG8, a novel small nucleolar guide RNA located on 4q26, was reported to have a high expression in EBV-associated GC [18]. Its precise biological role and mechanism of action in EBV-associated GC remain largely unclear.

Here, we explore the expression patterns of SNHG8 in EBV-associated GC and EBVnegative GC cell lines. We also examine the biological functions of SNHG8 in cell proliferation, cell cycle and apoptosis in vitro and in vivo.

\section{Methods}

\section{Cell lines and culture conditions}

Human gastric mucosal cell line GES-1 (Saierbio), EBV-associated GC cell lines GT38 and GT39 (American Type Culture Collection) and EBV-negative GC cell lines AGS and SGC7901 (Type Culture Collection of the Chinese Academy of Sciences) were used in this study. The cell lines were cultured in RPMI-1640 (Gibco; Thermo Fisher Scientific, Inc.) supplemented with 10\% fetal bovine serum (FBS; ExCell Bio), $50 \mathrm{U} / \mathrm{ml}$ penicillin $\mathrm{G}$ and $50 \mathrm{U} / \mathrm{ml}$ streptomycin (Gibco) in at $37{ }^{\circ} \mathrm{C}$ in a $5 \% \mathrm{CO}_{2}$ incubator. The medium was changed every 2 days and the cell line was passaged every 4 to 5 days.

\section{Cell transfection}

Cells were grown in monolayers and conventionally passaged when the cell attachment rate reached 90\%. The specific shRNA against SNHG8 was designed and synthesized at the Shanghai facility of Invitrogen. A negative control shRNA was synchronously synthesized. Cells were plated and cultured in growth media until the cell density reached $70 \%$. Then shRNA transfection was conducted with Lipofectamine 2000 reagent (Invitrogen) based on the manufacturer's protocol. Cells were harvested after $48 \mathrm{~h}$.

\section{Short hairpin RNA-expressing plasmid construction, lentivirus packaging, cloning and stable transfection}

To reduce the expression of SHNG8, human SHNG8 shRNA sequences were cloned into the pGIPZ-lentivirus vector (System Biosciences). Thereafter, SNHG8 knockdown vectors were constructed and sequenced. The empty pGIPZ vector without any insertion was used as a control. 293 T cells (Shanghai Research Institute of Chinese Academy of Sciences) were cultured in DMEM containing $10 \% \mathrm{FBS}$, maintained at $37{ }^{\circ} \mathrm{C}$ 
and transfected using Lipofectamine 2000 reagent with $3 \mu \mathrm{g}$ pGIPZ-SNHG8-shRNAs, $6.0 \mu \mathrm{g}$ PsPax 2 and $3 \mu \mathrm{g}$ pMD 2.G. The media were replaced with $10 \mathrm{ml}$ fresh medium after incubation overnight. The virus-containing supernatants (pGIPZ-neg-shRNA-LV and pGIPZ-SNHG8-shRNA-LV) were collected at $48 \mathrm{~h}$.

GT38 cells were infected and then selected using $4 \mu \mathrm{g} / \mathrm{ml}$ puromycin. The knockdown efficiency was measured using quantitative real-time PCR. Five days after infection, assays for cell proliferation, colony formation, cell cycle and cell apoptosis were performed.

In subsequent assays, the GT38 cells were divided into three groups in subsequent assays: blank control group (CON group; cells without infection), negative control (NC group; cells infected with pGIPZ-neg-shRNA-LV) and the SNHG8 knockdown group (KD group; cells infected with pGIPZ-SNHG8-shRNA-LV).

\section{Cell proliferation assay}

Cell proliferation was assessed using the 3-(4, 5-dimethyl-2-thiazolyl)-2, 5-diphenyltetrazolium bromide (MTT) assay as previously described [19]. Briefly, after stable depletion of SNHG8, GT38 cells were seeded in 96-well plates at a density of $5 \times 10^{3}$ cells/well and cultured for 1, 2 or 3 days. The supernatant was discarded, and $20 \mu \mathrm{l}$ of MTT was added for another $4 \mathrm{~h}$ of incubation in RPMI-1640 supplemented with 10\% FBS. Then, $150 \mu \mathrm{l}$ of dimethylsulfoxide (DMSO) was mixed with the cells for $10 \mathrm{~min}$. Absorbance of the cells in each well was observed at $570 \mathrm{~nm}$ under an Olympus absorption spectrophotometer for the cell number calculation. All the experiments were repeated three times.

\section{Clonogenic assay}

The clonogenic assay was performed with a modification of a previously published method. In brief, after completion of NC or specific shRNA transfection, GT38 cells were plated into 6-well plates in triplicate and at a cell density of 100 cells/well. Then, the cells were grown in RPMI-1640 containing 10\% FBS in an atmosphere of $5 \% \mathrm{CO}_{2}$ and $95 \%$ humidity at $37{ }^{\circ} \mathrm{C}$ for 14 days. After that, the cells were fixed and stained with crystal violet, followed by air-drying. Finally, the colonies were manually counted under an Olympus IX83 microscope.

\section{Cell apoptosis and the cell cycle assay}

After transfection with NC or specific shRNA, cells were cultured in RPMI-1640 containing 10\% FBS for another $48 \mathrm{~h}$. Then the cells were trypsinized and apoptosis was detected using an Annexin V-FITC Apoptosis Detection Kit (BD Biosciences). Then, cells were pelleted and washed with cold PBS and suspended in cold PBS. The cells were then treated with Annexin V-propidium iodide (PI) in the dark at room temperature according to the manufacturer's instructions. The cells were kept on ice in the dark and analyzed via flow cytometry using a FACSCalibur (BD Biosciences). The data were analyzed using Cell Quest software.

For cell cycle analysis, GT38 cells transfected with NC or specific shRNA were harvested after $48 \mathrm{~h}$. The cells were fixed with $70 \%$ ethanol at $-20{ }^{\circ} \mathrm{C}$ overnight and stained with PI (Sigma-Aldrich) in the presence of Ribonuclease A (Takara Biotechnology) for $30 \mathrm{~min}$ at room temperature. The cell cycle distribution was analyzed via flow 
cytometry using a FACSCalibur (BD Biosciences). All the experiments were repeated three times.

\section{Quantitative RT-PCR}

Total RNA was isolated from cell lines using TRIzol Reagent (Invitrogen). Two micrograms of total RNA were reverse transcribed to obtain cDNA using Moloney Murine Leukemia Virus Reverse Transcriptase (M-MLVRT; Progema) according to the manufacturer's instructions. Quantitative PCR was conducted with $1 \mu$ of cDNA using SYBR Green Taq Mix (Takara) on a Bio-Rad Real-Time PCR System. Glyceraldehyde 3-phophate dehydrogenase (GAPDH) was used as the internal control for normalization. The primers for SNHG8 were synthesized by Invitrogen. Their sequences were: SNHG8 primers, forward: 5'-AAGTTTACAAGCATGCGCGG-3'; reverse: 5' - TCAAACTGACGGTTCTCGGG-3'; GAPDH primers, forward: 5' - CGCTCTCTGCTCCTCCTGTTC-3'; reverse: 5'-ATCCG TTGACTCCG ACCTTCAC-3'.

The thermal cycling conditions were: $95{ }^{\circ} \mathrm{C}$ for 5 min, followed by 40 cycles of $95{ }^{\circ} \mathrm{C}$ for $30 \mathrm{~s}, 60{ }^{\circ} \mathrm{C}$ for $30 \mathrm{~s}$ and $72{ }^{\circ} \mathrm{C}$ for $1 \mathrm{~min}$. The final extension was $72{ }^{\circ} \mathrm{C}$ for $5 \mathrm{~min}$. Experiments were repeated at least three times and the relative expression of SNHG12 was calculated using $2^{-\triangle \Delta \mathrm{CT}}$ method.

\section{Animal experiments}

Five-week old male BALB/c nude mice were purchased from the Chinese National Rodent Laboratory Animal Resources. Animals were housed in a specific pathogen-free room in accordance with the current regulations and standards. All animals were allowed to acclimatize to their new environment for one week prior to use. Mice were randomly divided into 2 groups (6 mice/group): those receiving GT38 cells with SNHG8 depletion (shRNA) and the control group, which received non-transfected GT38 cells. For each group of mice, $5 \times 10^{6}$ cells/mouse were subcutaneously injected in the right side of the dorsal area.

Mice were then monitored for the growth of tumors. The tumor size was measured every 7 days for 28 days and estimated using the equation length $\times$ $(\text { width })^{2} \times 0.5$. After 30 days, the mice were killed, the tumors were removed, and the weight of each tumor was recorded. All animal experiments were performed according to the guidelines approved by the Chinese Association of Laboratory Animal Care.

\section{Statistical analysis}

All data in this study are expressed as means \pm SD, and differences between groups were determined using analysis of variance (ANOVA) with SPSS version 18.0. $p<0.05$ was considered statistically significant.

\section{Results}

\section{SNHG8 expression was upregulated in EBV-associated GC cells}

To investigate the roles of the lncRNA SNHG8 in EBV-associated GC pathogenesis, the expression of SNHG8 in EBV-associated GC cell lines was assessed using qRT-PCR (Fig. 1). The results show that the relative SNHG8 mRNA expression in 


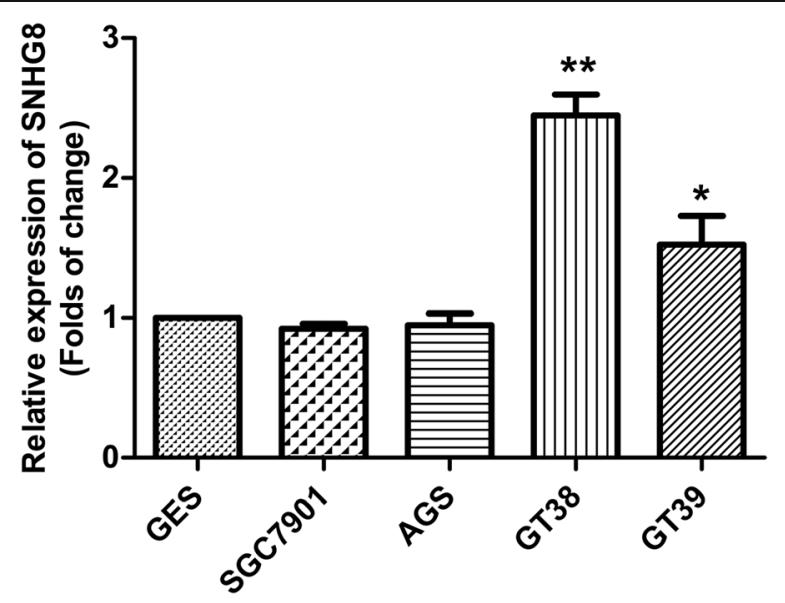

Fig. 1 LncRNA SNHG8 expression was upregulated in EBV-associated GC cells. The relative SNHG8 levels are shown for normal human gastric mucosal cell line GES-1, EBV-negative GC cell lines SGC7901 and AGS, EBVassociated GC cell lines GT38 and GT39. ${ }^{*} p<0.01$ and ${ }^{* *} p<0.01$ compared to GES-1.

GT38 and GT39 cells was significantly higher than that in the normal human gastric mucosal cell line GES-1 and the EBV-negative GC cell line AGS and SGC7901.

\section{Knockdown of SNHG8 impaired proliferation and colony formation of GT38 cells in vitro} As shown in Fig. 1, the SNHG8 expression in GT38 was higher than that of GT39. Therefore, we selected GT38 cell line as the model to investigate the effect of SNHG8 on cell proliferation and apoptosis.

A specific shRNA against SNHG8 was used to stably deplete SNHG8 expression. Transfection of a negative control (NC) or specific shRNA was performed, and $90 \%$ of the cells expressed green fluorescence protein, indicating successful transfection efficiency (data not shown).

The specific shRNA sh-SNHG8 significantly decreased the SNHG8 level by up to $57 \%$ in GT38 cells $(p<0.01$, Fig.2a). MTT assays showed that knockdown of SNHG8 obviously suppressed the proliferation rate of GT38 from day $2(p<0.01$, Fig. 2b). Consistent with these results, the ability to form colonies by GT38 cells was also suppressed significantly after knockdown of SNHG8 (Fig. 2c). Counting of colonies showed that colony numbers significantly decreased compared with the $\mathrm{NC}$ and control groups $(p<0.01$, Fig. $2 \mathrm{~d})$.

These results suggested that SNHG8 depletion has an inhibitory effect on the proliferation and clonogenic potential of EBV-associated GC cells.

\section{Knockdown of SNHG8 induced the apoptosis of GT38 cells}

To investigate the effect of SNHG8 on the cell apoptosis of EBV-associated GC cells, GT38 cells were stably transfected with the specific shRNAs (KD group) or without them (NC group), then stained with Annexin V and propidium iodide (PI), followed by detection using flow cytometry. As shown in Fig. 3, compared with the level for the NC group, the percentage of apoptosis in the KD group was 


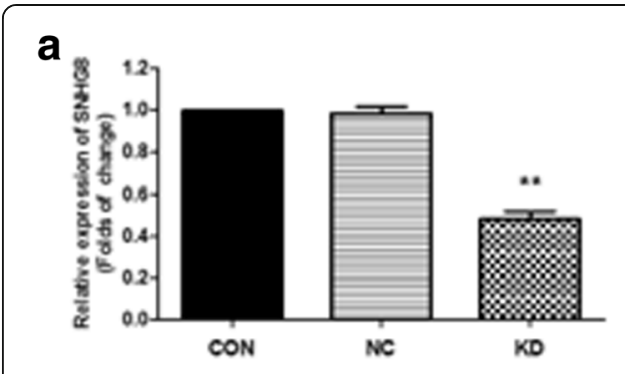

C

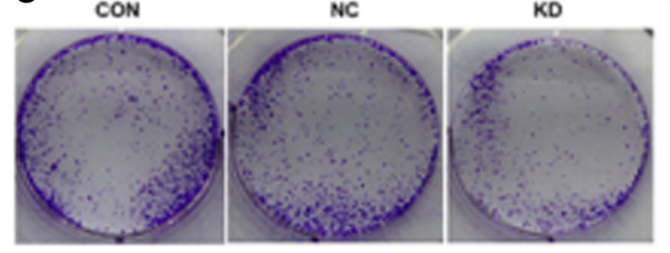

b

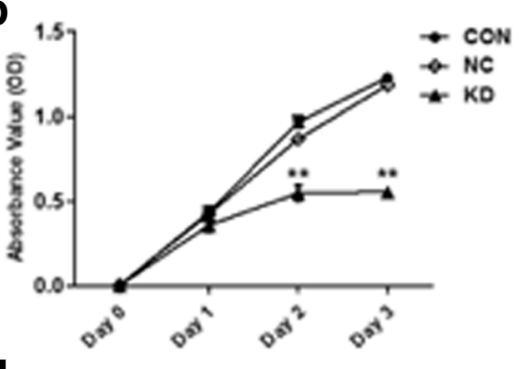

d

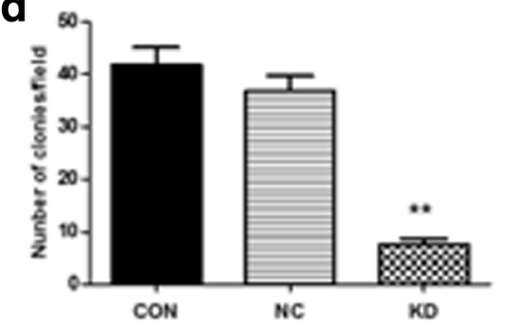

Fig. 2 Knockdown of SNHG8 inhibits cell proliferation and clonogenic potential in GT38 cells. a The specific shRNA sh-SNHG8 decreased the SNHG8 level by up to $57 \%$ in GT38 cells. ${ }^{* *} p<0.01$. b The results of 3 consecutive days of cell viability assessments for GT38 cells with (KD group) or without (NC group) specific shRNA transfections and blank control cells (CON group). c GT38 cells with (KD group) or without (NC group) specific shRNA transfections and blank control cells (CON group) were subjected to colony formation assays. After crystal violet staining, colonies were visually observed. $\mathbf{d}$ Counting of colonies showed that colony numbers had significantly decreased in the KD group. ${ }^{* *} p<0.01$ compared with NC group. CON, blank control cells without transfection; NC, negative control; KD, SNHG8 knockdown

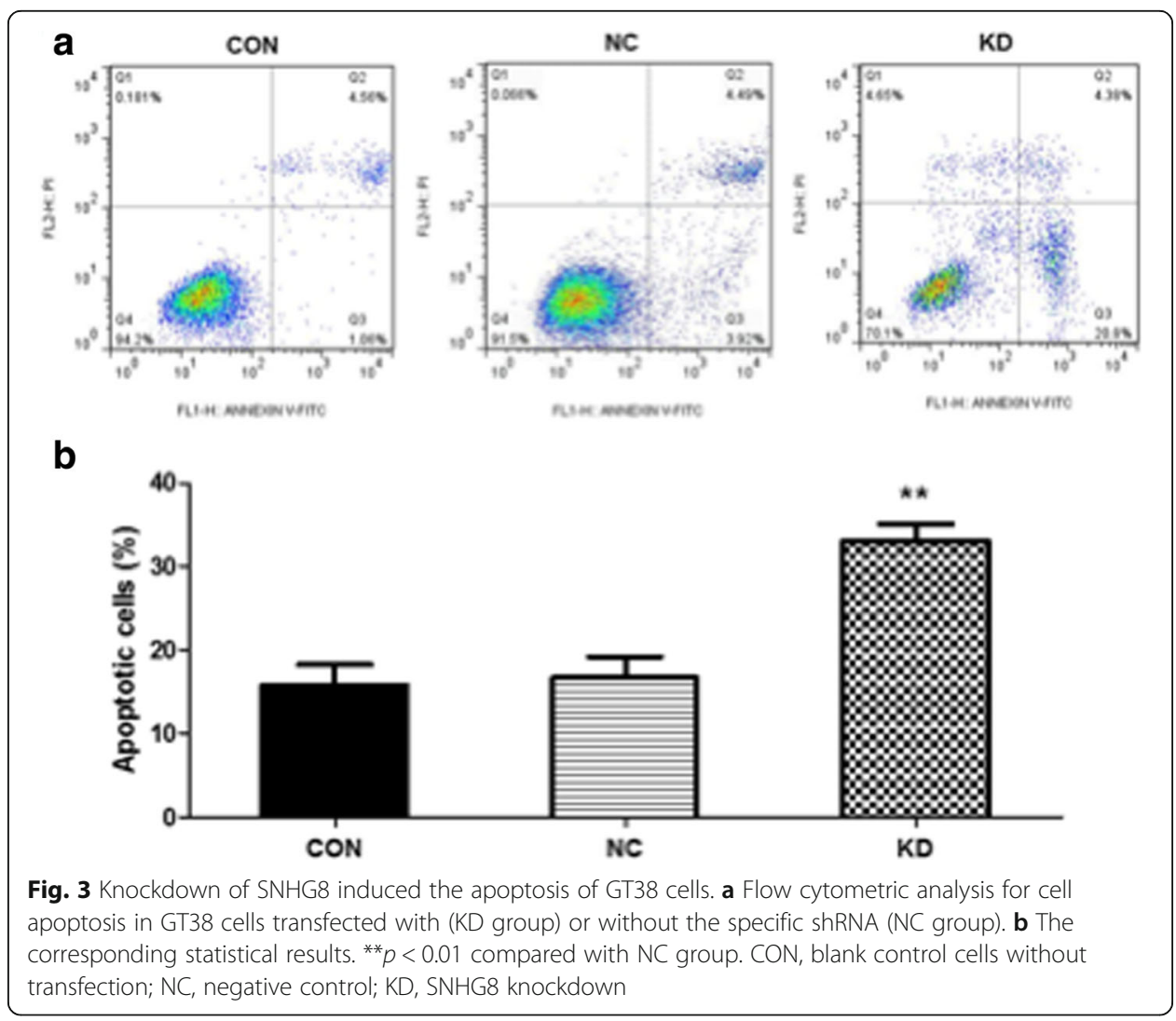


significantly higher. These results indicate that knockdown of SNHG8 induced cell apoptosis of GT38 cells.

\section{GT38 cells were arrested in G0/G1 phase after knockdown of SNHG8}

Having found the effect of SNHG8 downregulation on the proliferation of the EBVassociated GC cell line GT38, we then examined the impact of decreased expression of SNHG8 on the cell cycle. Flow cytometric analysis showed a decrease in the percentage of cells in S phase and a marked accumulation of cells in G0/G1 phase in the KD group of GT38 cells compared with the NC group (Fig. 4). Compared with the control group, a greater proportion of SNHG8 knockdown cells remained in G0/G1 phase while fewer cells were in S phase. These results indicate that downregulation of SNHG8 expression in GT38 cells arrested in G0/G1 phase.

\section{SNHG8 knockdown inhibited tumor growth in vivo}

To detect the effect of SNHG8 knockdown in vivo, a nude mouse xenograft model of EBV-associated GC cells was established. GT38 cells were stably transfected with shNC or sh-SNHG8 and subcutaneously injected into the nude mice $(n=6$ for each group). As shown in Fig. 5a and b, SNHG8 knockdown inhibited tumor growth significantly after 28 days. In addition, the tumor weight on day 28 in mice inoculated with GT38 cells transfected with sh-SNHG8 was significantly lower than that of the controls (transfected with sh-NC; Fig. 5c). These in vivo results indicate that SNHG8 knockdown inhibited tumor growth.

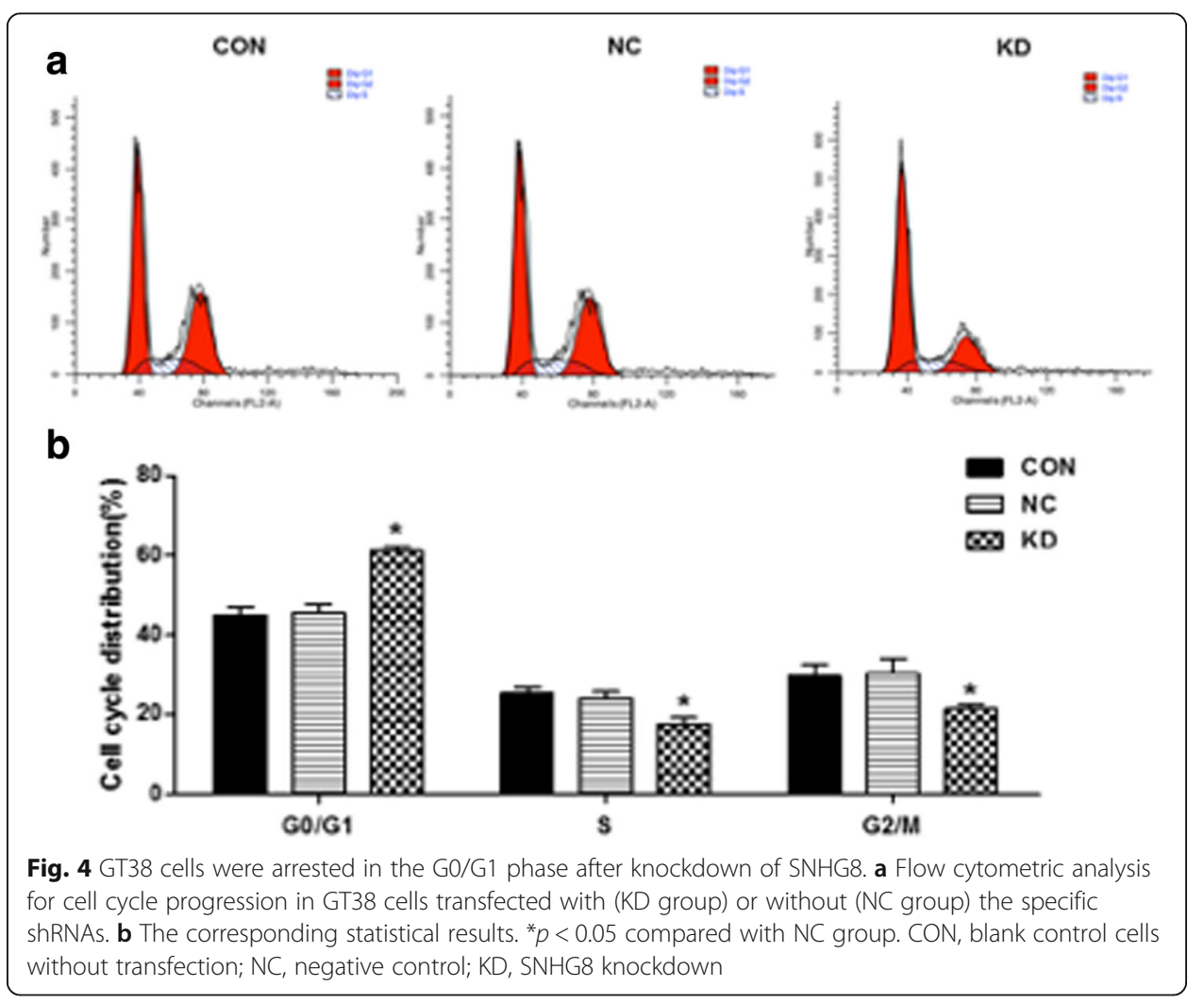




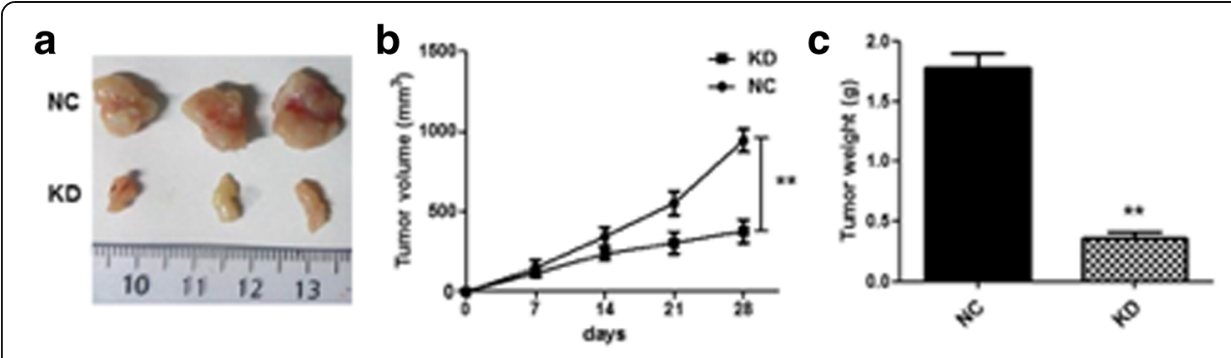

Fig. 5 Effect of SNHG8 knockdown on EBV-associated GC growth in vivo. A subcutaneous xenograft tumor model of GT38 cells in nude mice was established. GT38 cells were transfected with sh-NC (CON group) or shSNHG8 (KD group; $n=6$ ). a Tumors in mice inoculated with GT38 cells treated with sh-NC or sh-SNHG8. b Tumor size in the two groups. c Tumor weight in the two groups. ${ }^{* *} p<0.01$ compared with the CON group

\section{Discussion}

Gastric cancer (GC) is a complex and highly heterogeneous disease [20]. Epstein-Barr virus (EBV) is absent in non-cancerous mucosa but present in all cancer cells, and has a clonal nature in neoplastic cells; therefore, it is considered to have a causal role in gastric carcinoma [8, 21]. EBV-associated GC constitutes almost $10-18 \%$ of all GC cases. Due to the challenges in treating this cancer, identifying additional targets that could serve as biomarkers for early diagnosis and treatment is urgent.

LncRNAs are a large subgroup of non-coding transcripts that have emerged as crucial regulators and prognostic markers in multiple cancers, including GC. Researchers who are committed to the search for effective gastric cancer diagnostic markers have found that gastric cancer is associated with the dysregulation of specific lncRNAs. For example, overexpression of the lncRNA H19 promoted proliferation, migration, invasion and metastasis of GC [17]. Another study showed that SNHG5 was significantly downregulated and associated with the TNM stage in patients with GC [22].

Our study investigated the role of a newly identified lncRNA, SNHG8, in cell growth, cycle and apoptosis in EBV-associated GC using the GT-38 cell line. We found that SNHG8 expression was significantly upregulated in EBV-associated GC cell lines, SNHG8 depletion by specific shRNA slowed down proliferative rates in EBV-associated GC cells and clonogenic potential was consistently impaired after SNHG8 knockdown. We transfected the same SHNG8 shRNA into GT-39 cells, another EBV-associated GC cell, and found the same effect of SNHG8 in GT-39 cells (data not shown), proving that SNHG8 has the same importance in different EBV-associated GC cells.

A xenograft model of EBV-associated GC in nude mice further confirmed that SNHG8 knockdown inhibited tumor growth in vivo. All these data suggest a tumorigenic role of SNHG8 in EBV-associated GC. Furthermore, GT38 cells that were depleted of SNHG8 exhibited increased cell apoptosis. Cell cycle was arrested at G0/G1 stage when SNHG8 was silenced.

\section{Conclusions}

In summary, the findings from our study demonstrated that the downregulation of SNHG8 expression inhibits cell growth, arrests cell cycle and facilitates apoptosis. To the best of our knowledge, this study is the first to reveal a functional role for SNHG8 in EBV-associated GC. Using specific shRNA or developing novel targets against SNHG8 may be promising strategies for EBV-associated GC intervention or treatment. 


\section{Abbreviations}

DMSO: Dimethylsulfoxide; EBV: Epstein-Barr virus; FBS: Fetal bovine serum; GAPDH: Glyceraldehyde 3-phophate dehydrogenase; GC: Gastric cancer; IncRNAs: Long non-coding RNAs; MTT: 3-(4, 5-dimethyl-2-thiazolyl)-2, 5diphenyltetrazolium bromide; NPC: Nasopharyngeal carcinoma; ORFs: Open reading frames; PI: Propidium iodide; shRNA: Short hairpin RNA

\section{Acknowledgements}

Not applicable.

\section{Funding}

Not applicable.

\section{Availability of data and materials}

Not applicable.

\section{Authors' contributions}

All authors read and approved the final manuscript.

\section{Ethics approval}

All animal experiments in this study were performed according to the National Institutes of Health Guide for Care and Use of Laboratory Animals and approved by the institutional ethical committee.

\section{Consent for publication}

Not applicable.

\section{Competing interests}

The authors declare that they have no competing interests.

\section{Publisher's Note}

Springer Nature remains neutral with regard to jurisdictional claims in published maps and institutional affiliations.

\section{Author details}

${ }^{1}$ Department of Nephology, Zibo, China. ${ }^{2}$ Department of Gastrointestinal Surgery, Zibo, China. ${ }^{3}$ Department of Medical Care, Zibo Central Hospital, 54 Gongqingtuan Rd, Zibo, Shandong 255036, People's Republic of China.

Received: 20 November 2017 Accepted: 10 January 2018

Published online: 27 April 2018

\section{References}

1. Young LS, Rickinson AB. Epstein-Barr virus: 40 years on. Nat Rev Cancer. 2004;4:757-68.

2. He B, Li W, Wu Y, Wei F, Gong Z, Bo H, et al. Epstein-Barr virus-encoded miR-BART6-3p inhibits cancer cell metastasis and invasion by targeting long non-coding RNA LOC553103. Cell Death Dis. 2016;7:e2353.

3. Young LS, Murray PG. Epstein-Barr virus and oncogenesis: from latent genes to tumours. Oncogene. 2003;22: 5108-21.

4. Massini G, Siemer D, Hohaus S. EBV in Hodgkin Lymphoma. Mediterr J Hematol Infect Dis. 2009;1:e2009013.

5. He JR, Tang LY, Yu DD, Su FX, Song EW, Lin Y, et al. Epstein-Barr virus and breast cancer: serological study in a high-incidence area of nasopharyngeal carcinoma. Cancer Lett. 2011;309:128-36.

6. Rowe M, Fitzsimmons L, Bell Al. Epstein-Barr virus and Burkitt lymphoma. Chin J Cancer. 2014;33:609-19.

7. Nishikawa J, Yoshiyama H, lizasa H, Kanehiro Y, Nakamura M, Nishimura J, et al. Epstein-barr virus in gastric carcinoma. Cancers (Basel). 2014;6:2259-74.

8. Yao TO, Tang CM, Yu J. Epigenetic dysregulation in Epstein-Barr virus-associated gastric carcinoma: disease and treatments. World I Gastroenterol. 2014, 20:6448-56.

9. Siegel RL, Miller KD, Jemal A. Cancer Statistics, 2017. CA Cancer J Clin. 2017;67:7-30.

10. Digklia A, Wagner AD. Advanced gastric cancer: current treatment landscape and future perspectives. World J Gastroenterol. 2016;22:2403-14.

11. Kunej T, Obsteter J, Pogacar Z, Horvat S, Calin GA. The decalog of long non-coding RNA involvement in cancer diagnosis and monitoring. Crit Rev Clin Lab Sci. 2014;51:344-57.

12. Halley P, Kadakkuzha BM, Faghihi MA, Magistri M, Zeier Z, Khorkova O, et al. Regulation of the apolipoprotein gene cluster by a long noncoding RNA. Cell Rep. 2014;6:222-30.

13. Prensner JR, Iyer MK, Balbin OA, Dhanasekaran SM, Cao Q, Brenner JC, et al. Transcriptome sequencing across a prostate cancer cohort identifies PCAT-1, an unannotated lincRNA implicated in disease progression. Nat Biotechnol. 2011;29:742-9.

14. Hu JJ, Song W, Zhang SD, Shen XH, Qiu XM, Wu HZ, et al. HBX-upregulated IncRNA UCA1 promotes cell growth and tumorigenesis by recruiting EZH2 and repressing p27Kip1/CDK2 signaling. Sci Rep. 2016;6:23521.

15. Wang J, Qiu M, Xu Y, Li M, Dong G, Mao Q, et al. Long noncoding RNA CCAT2 correlates with smoking in esophageal squamous cell carcinoma. Tumour Biol. 2015;36:5523-8.

16. Ma P, Xu T, Huang M, Shu Y. Increased expression of LncRNA PANDAR predicts a poor prognosis in gastric cancer. Biomed Pharmacother. 2016;78:172-6.

17. Liu G, Xiang T, Wu QF, Wang WX. Long noncoding RNA H19-derived miR-675 enhances proliferation and invasion via RUNX1 in gastric cancer cells. Oncol Res. 2016;23:99-107. 
18. Huang $T$, Ji $Y$, Hu D, Chen B, Zhang H, Li C, et al. SNHG8 is identified as a key regulator of epstein-barr virus(EBV)associated gastric cancer by an integrative analysis of IncRNA and mRNA expression. Oncotarget. 2016;7:80990-1002.

19. Cohen MS, Hussain HB, Moley JF. Inhibition of medullary thyroid carcinoma cell proliferation and RET phosphorylation by tyrosine kinase inhibitors. Surgery 2002;132:960-966; discussion 6-7.

20. Torre LA, Bray F, Siegel RL, Ferlay J, Lortet-Tieulent J, Jemal A. Global cancer statistics, 2012. CA Cancer J Clin. 2015; 65:87-108.

21. Marquitz AR, Mathur A, Shair KH, Raab-Traub N. Infection of Epstein-Barr virus in a gastric carcinoma cell line induces anchorage independence and global changes in gene expression. Proc Natl Acad Sci U S A. 2012;109: 9593-8.

22. Zhao L, Guo H, Zhou B, Feng J, Li Y, Han T, et al. Long non-coding RNA SNHG5 suppresses gastric cancer progression by trapping MTA2 in the cytosol. Oncogene. 2016;35:5770-80.

Submit your next manuscript to BioMed Central and we will help you at every step:

- We accept pre-submission inquiries

- Our selector tool helps you to find the most relevant journal

- We provide round the clock customer support

- Convenient online submission

- Thorough peer review

- Inclusion in PubMed and all major indexing services

- Maximum visibility for your research

Submit your manuscript at www.biomedcentral.com/submit 